\title{
MODELLING THE DYNAMICS OF ABYSSAL EQUATOR-CROSSING CURRENTS
}

\author{
P.F. CHOBOTER AND G.E. SWATERS
}

\section{Introduction}

Abyssal flows, as part of the global thermohaline circulation, make a significant contribution to the flux of heat over the earth, and therefore affect the planet's climate. In the Atlantic, the deepest flow consists of Antarctic Bottom Water, which originates in the Weddell Sea near Antarctica. and flows northward along the western boundary of the Atlantic ocean. While part of this flow recirculates within the Brazil Basin, remaining in the southern hemisphere, part of the flow is observed to cross the equator into the northern hemisphere (DeMadron \& Weatherly, 1994; Friedrichs \& Hall, 1993).

Potential vorticity is conserved following the flow if friction effects are neglected. However, the fluid is relatively quiescent before and after crossing the equator, that is, planetary vorticity dominates relative vorticity. Therefore, since the planetary vorticity changes sign over the path of the flow, the potential vorticity of the fluid has also changed sign, and so is certainly not conserved! This violation of potential vorticity conservation in cross-equatorial flows and the breakdown of the geostrophic approximation at the equator constitute two significant challenges in modelling these flows.

We present a simplified model of large-scale flow across the equator. Edwards \& Pedlosky $(1998 a, b)$ show that the presence of friction in the dynamics is necessary for potential vorticity modification, and thus for cross-equatorial flow to exist. Additionally, Nof \& Borisov (1998; see also 
Borisov \& Nof, 1998) find that the geometry of the bottom topography plays a crucial role in the equator-crossing process. Accordingly, the model we study retains frictional and topographic effects. We compare the simplified model to the more sophisticated shallow water theory to identify to what extent the model captures the essential physics of the problem.

\section{Frictional geostrophic model}

One simple model which is geostrophic to leading order away from the equator, yet predicts well-defined velocities at the equator, may be written

$$
\begin{gathered}
-f v=-g^{\prime} \frac{\partial\left(h+h_{B}\right)}{\partial x}-r u \\
f u=-g^{\prime} \frac{\partial\left(h+h_{B}\right)}{\partial y}-r v \\
\frac{\partial h}{\partial t}+\nabla \cdot(h u)=0
\end{gathered}
$$

where $\boldsymbol{u}=(u, v)$ is the horizontal velocity, $h$ is the height of the fluid layer, $h_{B}$ is the bottom topography elevation, $g^{\prime}$ is the reduced gravity, $f$ is the Coriolis parameter, and $r$ is a small damping coefficient to be specified. Note that this model does, in fact, retain the effects of an arbitrary bottom topography and parameterizes the effects of friction.

Models in which the momentum equations have been reduced to the geostrophic relations with the addition of a linear term representing the effects of friction have been used recently to study large-scale motions by several authors (see Stephens \& Marshall 2000; Edwards, Willmott \& Killworth 1998; Samelson 1998; Samelson \& Vallis 1997; and further references therein). In particular, Stephens \& Marshall (2000) numerically integrate a similar model over realistic bottom topography out to steady state in order to model the path of Antarctic Bottom Water across the equator. The resulting steady flow is found to be broadly consistent with observations.

In this model, the velocities may be solved for in a diagnostic relation in terms of the pressure gradients,

$$
u=g^{\prime} \frac{-f p_{y}-r p_{x}}{f^{2}+r^{2}}, \quad v=g^{\prime} \frac{f p_{x}-r p_{y}}{f^{2}+r^{2}}
$$

where $p=h+h_{B}$ and subscripts denote partial derivatives. Thus, the model contains a geostrophic component (terms proportional to $f$ in the numerator), and a down-pressure-gradient component (terms proportional to $r$ in the numerator). In the limit as $f \rightarrow 0$, the motion is that of a potential flow. 
By substituting the velocity relations (4) into the conservation of mass equation (3), a single evolution equation for the height field may be written

$$
h_{t}+J\left(g^{\prime}\left(h+h_{B}\right), \frac{h \dot{f}}{f^{2}+r^{2}}\right)=r \nabla \cdot\left[\frac{g^{\prime} h \nabla\left(h+h_{B}\right)}{f^{2}+r^{2}}\right],
$$

where $J(A, B)=A_{x} B_{y}-A_{y} B_{x}$. In this form, the model is clearly nonlinear and diffusive, with the amount of diffusion controlled by the parameter $r$.

The potential vorticity equation of this model is

$$
\frac{\partial}{\partial t}\left(\frac{f}{h}\right)+u \cdot \nabla\left(\frac{f}{h}\right)=-\frac{r}{h} \zeta
$$

where $\zeta=v_{x}-u_{y}$ is the relative vorticity. This model, then, effectively neglects relative vorticity in favour of planetary vorticity, and has the feature that it simulates the dissipation of potential vorticity by Ekman friction.

The major disadvantage of this model is its oversimplification of the dynamics. In particular, fluid inertia has been neglected. Since the fluid must always move down the pressure gradient, a mass of fluid flowing down one side of a valley does not have the momentum to flow back up the other side.

\section{Frictional geostrophic versus shallow water}

\subsection{SCALINGS}

We numerically integrate forward in time the reduced-gravity shallow water model and the frictional geostrophic model in order to compare the two models. The shallow water model may be written in non-dimensional form as

$$
\begin{gathered}
\frac{\partial \boldsymbol{u}}{\partial t}+\boldsymbol{u} \cdot \nabla \boldsymbol{u}+\frac{f}{R o} \mathbf{k} \times \boldsymbol{u}=-\frac{1}{R o} \nabla\left(h+h_{B}\right)+\boldsymbol{F}_{\text {fric }} \\
\frac{\partial h}{\partial t}+\nabla \cdot(\boldsymbol{u} h)=0
\end{gathered}
$$

where $\boldsymbol{u}$ is the horizontal velocity vector, $\boldsymbol{F}_{\boldsymbol{f} \text { ric }}$ represents the friction term, $R o=U / f_{0} L$ is the Rossby number, and $U, L, f_{0}$, and $h_{0}$ are typical scales for the velocity, length, Coriolis parameter and fluid depth. It has been assumed that the time variable is scaled advectively, $T=L / U$, for a time scale $T$, and that the scale slope for the bottom topography is the same as the scale slope of the fluid height, $h_{0} / L$. We have also employed the geostrophic scaling $U^{2} /\left(g^{\prime} h_{0}\right)=R o$. Since $f$ passes through zero in the domain of interest, $f_{0}$ is taken to be the maximum dimensional value of $f$ in the domain. It is assumed that the flow is geostrophic at that latitude. 
The numerical methods used are based upon the methods of Hallberg $\&$ Rhines (1996). For brevity, the details are not reported here, but may be found in Choboter \& Swaters (2000).

The simple model and the shallow water model are compared for flow over simplified bottom topography. The topography takes the shape of a meridional channel. Simulations were performed with the fluid initially south of the equator, flowing northward along the western half of the channel, in the form of an eddy, i.e. the height field initially has compact support in the domain. These initial conditions were chosen, in part, to simulate the Antarctic Bottom Water flow, which flows northward along the western slope toward the equator.

The bottom topography and functional form of Coriolis parameter are chosen in a particular way to provide a clean testing ground for the comparison of the two models. In particular, we are interested in diagnosing how well the propagation speed of the eddy agrees with the Nof (1983) speed, $g^{\prime} s / f$, where $s$ is the bottom slope. A nearly constant bottom slope and Coriolis parameter away from the channel bottom and equator facilitates computing this diagnostic. Therefore, the bottom topography is chosen to be a simplified meridional channel of hyperbolic cross section, $h_{B}=\sqrt{x^{2}+1}$, which has a slope approaching \pm 1 away from $x=0$, and the Coriolis parameter is chosen to be $f=\tanh \left(\beta_{0} L y / f_{0}\right)$, which tends to a non-dimensional $f$-plane value of unity away from $y=0$, and has a slope at $y=0$ of $\beta_{0} L / f_{0}$. For simulations reported here, $\beta_{0} L / f_{0}=1$, which, for $f_{0}$ evaluated at $5^{\circ}$ latitude, corresponds to choosing a horizontal length scale of $L=500 \mathrm{~km}$.

\subsection{RESULTS}

Several simulations of an isolated abyssal dome of fluid approaching the equator from the south have been carried out varying only the damping parameter $r$ in the case of the simple model, or the Rossby number $R o$ in the case of the shallow water model. In figure 1, we show snapshots from a typical simulation employing the shallow water equations. The eddy is observed to propagate along the shelf without losing much height until almost at the equator, when fluid starts to accelerate downhill. Part of the fluid is located slightly north of the equator while flowing downhill. The fluid rises up the other side of the channel, and ultimately splits into two eddies, one flowing north and one flowing south. This is qualitatively consistent with the simulations of Borisov \& Nof (1998), who investigated eddies crossing the equator in a meridional channel.

Figure 2 displays the simulation of the motion of the same initial eddy, but as predicted by the simple model. The eddy is seen to initially travel 


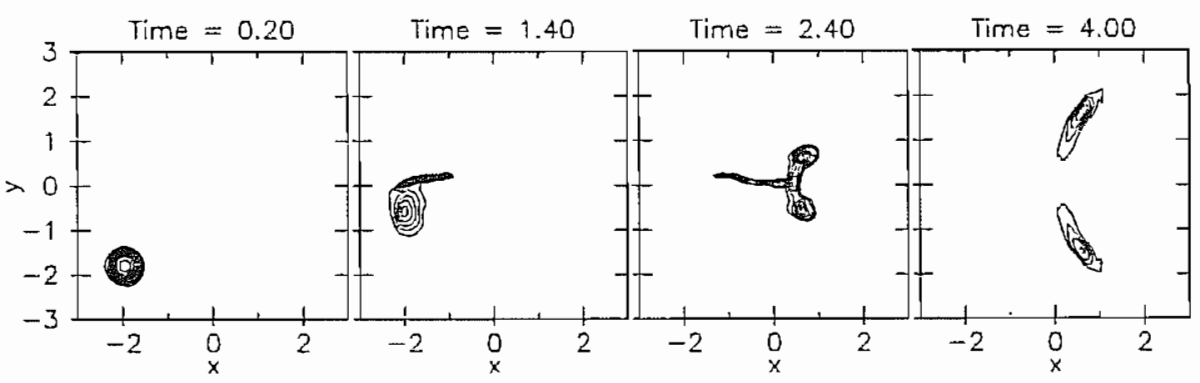

Figure 1. The results of a shallow water simulation, $R o=0.02$ The contour spacing is 0.02 .

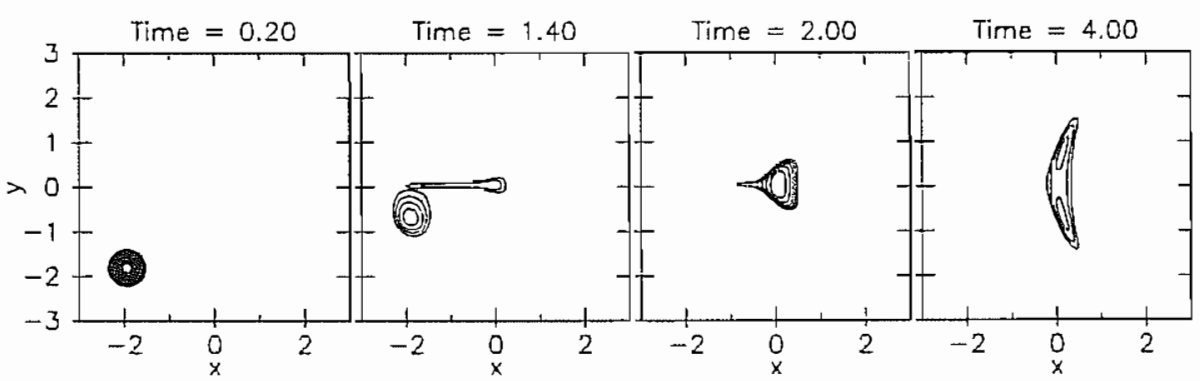

Figure 2. The results of a frictional geostrophic simulation, $r=0.02$. The contour spacing is 0.02 .

along the slope, as in the shallow water simulation, but upon reaching the equator, flows directly downhill, with very little fluid found north of the equator as it does so. The fluid pools at the bottom of the channel at the equator, and then proceeds to split into two parts, one flowing north, and the other recirculating back south. Despite the simplicity of the model, it captures the characteristic splitting of the fluid into northward and southward flowing parts seen in the shallow water simulation. The lack of inertia in the model is seen in both the sharp turn from along-slope flow to downhill flow and the immediate deceleration from fast downhill flow to nearly stationary fluid pooling at the equatorial channel bottom. Thus the net result of the lack of inertia in the model is that the north-south splitting of the flow is very symmetric, and that the final flow is very near the bottom of the channel.

Further analysis of this model (Choboter \& Swaters 2000) shows that the simple model simulations capture well the along-shelf Nof (1983) speed. For all the simple model runs, the fluid does not flow as high onto the opposite bank as for the shallow water runs, and a very symmetric northsouth splitting of the fluid is predicted. This further points to the lack of fluid inertia in the simple model. 


\section{Concluding remarks}

The model studied here parameterizes frictional and other ageostrophic effects into a simple Rayleigh damping term. We have investigated the viability of this model by comparing its predictions to the predictions of shallow water theory. Despite the simplicity of the model, it broadly captures certain aspects of shallow water flow quite well, such as the Nof (1983) along-slope eddy speed and the north-south splitting of the fluid. However, the model neglects the inertia of the fluid, which restricts the motion.

The bottom topography of the Atlantic Ocean is certainly more complicated than a meridional channel. It remains for future research to compare the predictions of these models over more realistic topography.

Preparation of this paper was supported by a Killam Postgraduate Scholarship awarded to P.F.C. and a research grant awarded to G.E.S. by the Natural Sciences and Engineering Research Council.

\section{References}

Borisov, S. \& Nof, D. (1998) Deep, cross-equatorial eddies. Geophys. Astrophys. Fluid Dyn. 87, 273-310.

Choboter, P. F. \& Swaters, G. E. (2000) Modelling equator-crossing currents on the ocean bottom. Submitted to Can. App. Math. Quart.

DeMadron, X. D. \& Weatherly, G. (1994) Girculation, transport and bottom boundary layers of the deep currents in the Brazil Basin. J. Mor. Res. 52, 583-638.

Edwards, N. R., Willmott, A. J. \& Killworth, P. D. (1998) On the role of topography and wind stress on the stability of the thermohaline circulation. J. Phys. Occanogr. 28, $756-778$.

Edwards, C. A. \& Pedlosky, J. (1998a) Dynamics of nonlinear cross-equatorial flow. Part I: Potential vorticity transformation. J. Phys. Oceanogr. 28, 2382-2406.

Edwards, C. A. \& Pedlosky, J. (1998b) Dynamics of nonlinear cross-equatorial flow. Part II: The tropically enhanced instability of the western boundary current. J. Phys. Oceanogr. 28, 2407-2417.

Friedrichs, M. A. M. \& Hall, M. M. (1993) Deep circulation in the tropical North Atlantic. J. Mar. Res. 51, 697-736.

Hallberg, R. \& Rhines, P. (1996) Buoyancy-driven circulation in an ocean basin with isopycnals intersecting the sloping boundary. J. Phys. Oceanogr. 26, 913-940.

Nof, D. (1983) The translation of isolated cold eddies an a sloping bottom. Deep-Sea Res. 30, 171-182.

Nof, D. \& Borisov, S. (1998) Inter-hemispheric oceanic exchange. Q. J. R. Meteorol. Soc. 124, 2829-2866.

Samelson, R. M. (1998) Large-scale circulation with locally enhanced vertical mixing. J. Phys. Oceanogr. 28, 712-726.

Samelson, R. M. \& Vallis, G. K. (1997) A simple friction and diffusion scheme for planetary geostrophic basin models. J. Phys. Oceanogr. 27, 186-194.

Stephens, J. C. \& Marshall, D. P. (2000) Dynamical pathways of Antarctic Bottom Water in the Atlantic. J. Phys. Oceanogr. 30, 622-640. 